\title{
Single-species fragmentation: The role of density-dependent feedback
}

\author{
V. Dornelas, ${ }^{1,}{ }^{*}$ E. H. Colombo, ${ }^{2}$ and C. Anteneodo ${ }^{1,3}$ \\ ${ }^{1}$ Department of Physics, PUC-Rio, Rua Marquês de São Vicente, 225, 22451-900, Rio de Janeiro, Brazil \\ ${ }^{2}$ IFISC (CSIC-UIB), Campus Universitat Illes Balears, 07122, Palma de Mallorca, Spain \\ ${ }^{3}$ Institute of Science and Technology for Complex Systems, Rio de Janeiro, Brazil
}

(Received 22 March 2019; published 26 June 2019)

\begin{abstract}
Internal feedback is commonly present in biological populations and can play a crucial role in the emergence of collective behavior. To describe the temporal evolution of the distribution of a single-species population, we consider a generalization of the Fisher-KPP equation. This equation includes the elementary processes of random motion, reproduction, and, importantly, nonlocal interspecific competition, which introduces a spatial scale of interaction. In addition, we take into account feedback mechanisms in diffusion and growth processes, mimicked by power-law density dependencies. This feedback includes, for instance, anomalous diffusion, reaction to overcrowding or to the rarefaction of the population, as well as Allee-like effects. We show that, depending on the kind of feedback that takes place, the population can self-organize splitting into disconnected subpopulations, in the absence of external constraints. Through extensive numerical simulations, we investigate the temporal evolution and the characteristics of the stationary population distribution in the one-dimensional case. We discuss the crucial role that density-dependence has on pattern formation, particularly on fragmentation, which can bring important consequences to processes such as epidemic spread and speciation.
\end{abstract}

DOI: 10.1103/PhysRevE.99.062225

\section{INTRODUCTION}

Population fragmentation is characterized by critical changes in the spatial distribution of individuals, creating isolated subgroups of a given initial population. This phenomenon has important consequences for secondary processes such as epidemic spreading, species invasion [1], or also speciation [2]. Fragmentation is often attributed to landscape heterogeneity, which encompasses the spatial distribution of geographic and environmental features [3]. As a matter of fact, if natural barriers are sustained for long periods of time, fragmentation can be induced [2].

This scenario has been extensively studied in the context of metapopulation theory, which at its core incorporates landscape heterogeneity [4]. The role played by the degree of fragmentation of the landscape, which is imposed on the population, is well known, determining the richness of species and stability of the ecosystem against external disturbances [4-6]. But regardless of environmental heterogeneity, the arrangements of individuals in space can emerge solely from their interactions, bringing critical consequences to the evolutionary dynamics and social behavior of living organisms [7-11].

We explore in this work under which conditions the population dynamics can self-induce fragmentation in the absence of external barriers. An earlier study pointed out that spatial patterns in population distribution may become disconnected when individuals' dispersal is subdiffusive [12]. We extend that investigation, deepening the characterization of the fragmentation process and assuming a more general nonlinear dynamics, where both dispersal and growth can be regulated

*vivian@aluno.puc-rio.br by the population concentration. In this way, we generalize the well-known Fisher-KPP equation [13,14], which relies on standard diffusion and logistic growth.

Density-dependent mobility may arise due to the structure of the environment $[15,16]$, but may also arise from complex biological and social responses to either overcrowding or rarefaction of population density [12,17-23]. For example, in insect populations, it has been observed that the diffusion coefficient can be enhanced or reduced by the population concentration [18]. In this and many other examples [18,24-27], a power-law diffusion coefficient was used as a phenomenological description.

Population growth can also be governed by densitydependent factors [27-33]. For instance, related to the Allee effect [34], the per capita reproduction rate vanishes at the low concentration limit. But there are also cases where reproduction is favored when the concentration is low, due to the absence of overpopulation disadvantages [35,36].

In addition to all this, our model considers the sharing of resources within a given spatial range, through a nonlocal competition term. In vegetation, for instance, long roots can induce water competition at a distance [37-39]. The release of toxic substances into the environment can also promote death at spatial scales much larger than the size of the individual [40]. Such mechanisms generate an effective kernel, also known as an influence function, which introduces a distance-dependent spatial coupling [41]. Under some conditions, such spatial coupling may promote spatial instability, a key ingredient for pattern formation [41-43].

It is worth noting that our modeling based on the FisherKPP equation is intended to describe the temporal evolution of population distributions, but it may also describe gene distribution or niche occupation [13]. So the fragmentation process 
that we focus in this work has an interesting ambiguity, which can be translated into speciation $[43,44]$.

The paper is organized as follows. In Sec. II we define the generalization of the Fisher-KPP equation that we use as a paradigmatic model. In Sec. III we obtain analytical results to define the conditions for pattern formation, and in Sec. IV we present the main results of numerical simulations, aiming to characterize the different classes of patterns, particularly the fragmented ones. In Sec. V a summary and discussion of the main results and possible extensions are presented.

\section{MODEL}

We consider the following generalization of the onedimensional Fisher-KPP equation [13] for the spatial distribution of one-species populations:

$\partial_{t} \rho(x, t)=\partial_{x}\left(D(\rho) \partial_{x} \rho\right)+f(\rho) \rho-b \rho \int_{-\infty}^{\infty} \gamma(x-y) \rho(y) d y$.

The first term on the right side of Eq. (2.1) corresponds to nonlinear diffusion, where the diffusion coefficient $D(\rho)$ depends on the local density $\rho(x, t)$. The second term regulates reproduction, which occurs with the per capita growth rate $f(\rho)$, which also depends on the local density. The last term represents nonlocal intraspecific competition, where $b>0$, and the (normalized) influence function $\gamma$ sets how the interaction depends on distance.

Following the motivations given in the Introduction, we investigate the class of dynamics where diffusion and growth coefficients have power-law density dependencies:

$$
\begin{gathered}
D(\rho)=D \rho^{\nu-1}, \\
f(\rho)=a \rho^{\mu-1},
\end{gathered}
$$

where $D, a, v$, and $\mu$ are positive parameters. For a logistic effect (referring to limited resources), we must have $\mu<2$, to ensure that the population size remains bounded.

Before proceeding, we nondimensionalize Eq. (2.1), by defining the scaled variables

$$
\begin{aligned}
\rho^{\prime} & =\rho / \rho_{0}, \\
t^{\prime} & =a \rho_{0}^{\mu-1} t, \\
x^{\prime} & =\sqrt{a \rho_{0}^{\mu-v} / D} x,
\end{aligned}
$$

where $\rho_{0}=(b / a)^{1 /(\mu-2)}$ is the uniform stationary solution, which becomes $\rho_{0}^{\prime}=1$. Then, substituting the scaling relations (2.4) into Eq. (2.1) and eliminating the prime superindexes, Eq. (2.1) becomes

$$
\partial_{t} \rho(x, t)=\partial_{x}\left(\rho^{\nu-1} \partial_{x} \rho\right)+\rho^{\mu}-\rho \int_{-\infty}^{\infty} \gamma(x-y) \rho(y) d y .
$$

Thus, $\mu$ and $v$ exponents are the only remaining parameters, once the kernel $\gamma$ is fixed.

\section{LINEAR STABILITY ANALYSIS}

Following the standard procedure, we assume a small perturbation around the nontrivial homogeneous steady state, $\rho(x, t)=1+\varepsilon(x, t)$, that we introduce into Eq. (2.5), obtaining

$$
\begin{aligned}
\partial_{t} \varepsilon= & \partial_{x}\left[(1+\varepsilon)^{\nu-1} \partial_{x} \varepsilon\right]+(1+\varepsilon)^{\mu} \\
& -(1+\varepsilon) \int_{-\infty}^{\infty} \gamma(x-y)[1+\varepsilon] d y .
\end{aligned}
$$

Keeping only terms up to first order in $\varepsilon$ and recalling that $\gamma$ is normalized, we obtain

$$
\partial_{t} \varepsilon=\partial_{x x} \varepsilon+(\mu-1) \varepsilon-\int_{-\infty}^{\infty} \gamma(x-y) \varepsilon d y,
$$

which in Fourier space becomes

$$
\partial_{t} \tilde{\varepsilon}(k, t)=\lambda(k) \tilde{\varepsilon}(k, t),
$$

where the tilde indicates a Fourier transform, and the rate $\lambda(k)$ is given by the dispersion relation

$$
\lambda(k)=-k^{2}-\tilde{\gamma}(k)+\mu-1 .
$$

Pattern formation occurs when there is a certain dominant mode $k^{\star}$ that stands out in the dispersion relation, that is, yielding the maximum positive rate $\lambda\left(k^{\star}\right)$ [45]. The condition for pattern formation $\left(\lambda\left(k^{\star}\right)>0\right)$ depends on the profile of the influence function $\gamma$ that must introduce a well-defined spatial scale of interaction [43]. The simplest form that verifies this property, promoting spatial instability, is the homogeneous influence function, which is constant inside a given region of width $2 \ell$,

$$
\gamma(x-y)=\frac{1}{2 \ell} \Theta(\ell-|x-y|),
$$

where $\Theta$ is the Heaviside function, being nonnull only if $|x-y|<\ell$. Therefore, the kernel Fourier transform is

$$
\tilde{\gamma}(k)=\sin (k \ell) /(k \ell) \text {. }
$$

The first term in Eq. (3.4), associated with diffusion, is always negative with maximum at $k=0$, tending to stabilize the homogeneous state. The term $\tilde{\gamma}(k)$ given by Eq. (3.6), associated with nonlocality, takes positive values that contribute to destabilize the homogeneous state and the maximum is located at $k \neq 0$, conditions that give rise to pattern formation. In addition, the nonlinearity $\mu \neq 1$ shifts the dispersion relation upwards with respect to the linear case $(\mu=1)$, contributing in favor of de(stabilization) when $\mu>1(\mu<1)$. Notice that the diffusion exponent $v$ does not appear explicitly in the dispersion relation.

The dominant mode $k^{\star}$, which is the maximum of $\lambda(k)$, can be approximated by $k^{\star} \ell \simeq 3 \pi / 2$ [12]. Its rate of exponential change is positive if

$$
\mu>\mu_{p} \equiv\left(k^{\star}\right)^{2}-\frac{1}{k^{\star} \ell}+1
$$

This is the frontier for the onset of patterns. Moreover, when patterns appear, the number $m$ of peaks can be estimated by

$$
m=\frac{k^{\star} L}{2 \pi} \simeq 0.715 \frac{L}{\ell},
$$

where $L$ is the system size.

Note that nonlinearities are also contained in the time and space scales, according to Eqs. (2.4), so they influence the growth rate of the instability and the pattern wavelength. 

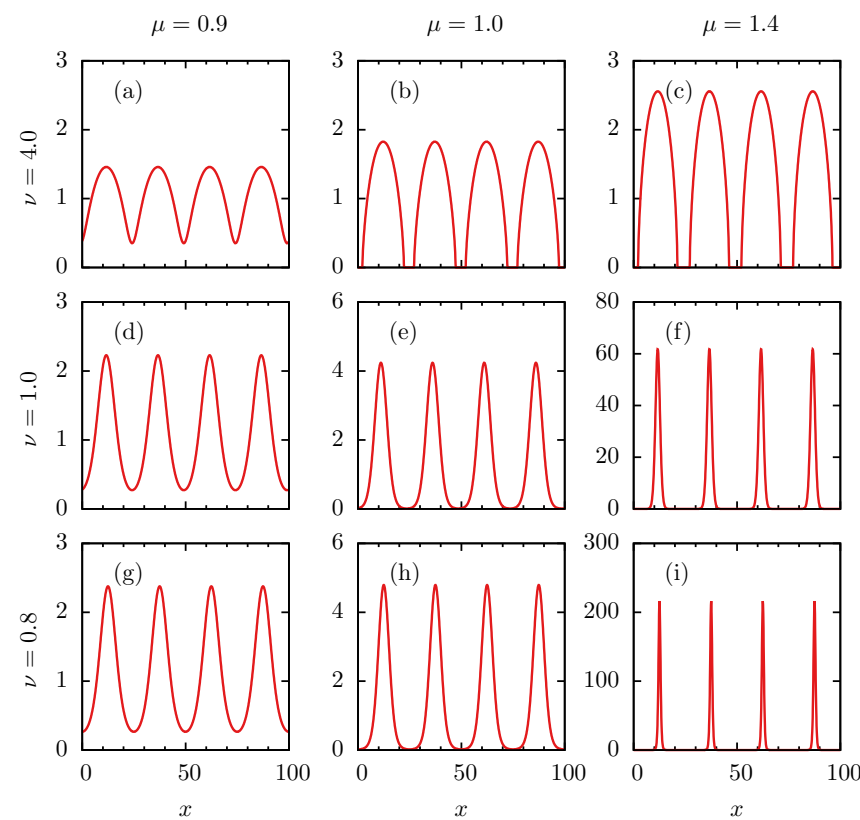

FIG. 1. Stationary profiles $\rho(x)$ for different values of $v$ : 0.8 (superdiffusion), 1.0 (normal diffusion), and 4.0 (subdiffusion), and different values of the growth exponent $\mu: 0.9,1.0$, and 1.4.

Therefore, although $v$ does not appear explicitly in Eq. (3.4), it has an indirect influence.

\section{NUMERICAL RESULTS}

Numerical integration of Eq. (2.5) was performed using a forward-time, centered-space scheme [46], considering a one-dimensional domain with periodic boundary conditions. Starting from the homogeneous steady state $\rho_{0}=1$, with the addition of a white-noise perturbation, uniform in $\left[-\delta \rho_{0}, \delta \rho_{0}\right]$ with $\delta \rho_{0}=10^{-2}$, we let the dynamics evolve until the stationary regime has been reached. In simulations, we used space and time increments, $d x \leqslant 10^{-1}$ and $d t \leqslant 10^{-4}$, respectively [47].

In all numerical simulations, we set the system size $L=$ 100 and the competition interaction range $\ell=20$. As a consequence of this choice, Eq. (3.8) predicts that, when there are patterns (i.e., when $\mu>\mu_{p} \simeq 0.84$ ), the estimated number $m$ of peaks is $m=3.75$. Therefore, more likely we observe four peaks.

Typical profiles that emerge in our numerical simulations are presented in Fig. 1, for different values of $v$ and $\mu$ in the region where $\lambda(k)>0$ (see also the Appendix for further details). In the standard case $\mu=v=1$, each individual peak has a Gaussian-like shape. But when feedback is taken into account, mobility and reproduction rates respond to the degree of agglomeration of individuals. Then the peaks tend to be more platykurtic (leptokurtic) when $v>1(v<1)$, since the diffusion rate vanishes (diverges) at low densities. With respect to the exponent $\mu$, it is evident that the patterns that emerge when $\mu<1$ have a minimum value which is noticeably nonzero, in contrast to the cases $\mu \geqslant 1$. These traits can be associated with the type of density-dependent feedback (governed by $\mu$ ): when $\mu<1$, population growth

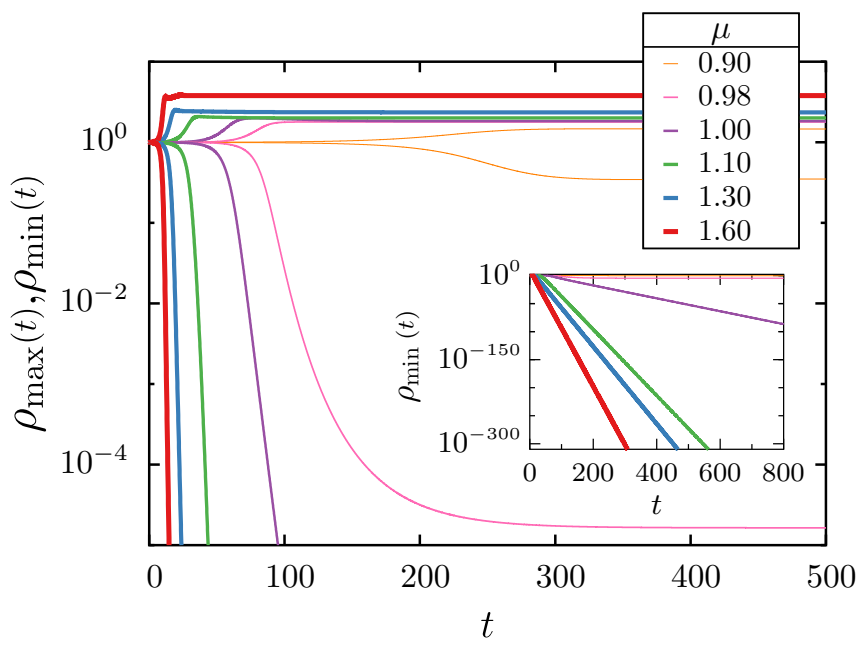

FIG. 2. Temporal evolution of the maximal and minimal densities $\rho_{\max }(t)$ and $\rho_{\min }(t)$, for $\nu=4.0$ and values of $\mu$ indicated in the legend (thicker lines correspond to larger values of $\mu$ ). Inset: $\rho_{\min }(t)$ on a larger scale.

is enhanced in low-density regions, raising the population level in the valley between clusters; while for $\mu>1$, the opposite effect occurs. The combination of diffusion and growth nonlinearities generates the diverse profiles shown in Fig. 1. Next, we will discuss the emergence of these profiles, focusing on the characterization and definition of fragmented states [Figs. 1(b) and 1(c)].

To identify the fragmentation process, we follow the temporal evolution of the smallest value of the concentration of individuals, $\rho_{\min }(t)$. Representative cases are shown in Fig. 2, where in addition to the minimal value, also the largest value $\rho_{\text {max }}(t)$ is plotted.

We note that for small enough values of $\mu, \rho_{\min }(t)$ stabilizes at a finite level. In contrast, for $\mu$ greater than a critical value ( $\mu_{c} \simeq 1$, in the case of Fig. 2), $\rho_{\min }(t) \sim \exp (-t / \tau)$, decreasing exponentially with time to the computational limit $\left(\varrho \sim 10^{-308}\right)$. The characteristic time $\tau$ is represented in Fig. 3, as a function of the exponents, including the cases shown in Fig. 2.

The numerical outcomes suggest the emergence of disconnected clusters, separated by depopulated regions, when $\nu$ and $\mu$ obey certain conditions. To better characterize the fragmented patterns and the conditions for their emergence, we consider in addition to the stationary values $\left(\rho_{\max }\right.$ and $\left.\rho_{\text {min }}\right)$, the width $\sigma$ of each cluster at half height and the length $\Delta$ of the region where $\rho$ attains $\varrho$, which we interpret as null density [48]. The results are shown in Fig. 4, varying the diffusion exponent $v$ while keeping the growth exponent $\mu$ constant. For $\mu=0.9$ [Fig. 4(a)], the shape of the patterns is almost insensitive to $v$. Importantly, we do not detect a region where the density vanishes (for this reason, $\Delta$ symbols are absent in the plot); that is, fragmentation does not occur. Differently, in Figs. 4(b) and 4(c), a sharp drop of $\rho_{\min }$ is observed as a function of $\mu$. Concurrently, a nonzero $\Delta$ is detectable in these cases. In this sense, patterns become fragmented, which occurs beyond a critical value of $v$ (which decreases with $\mu$ ). 

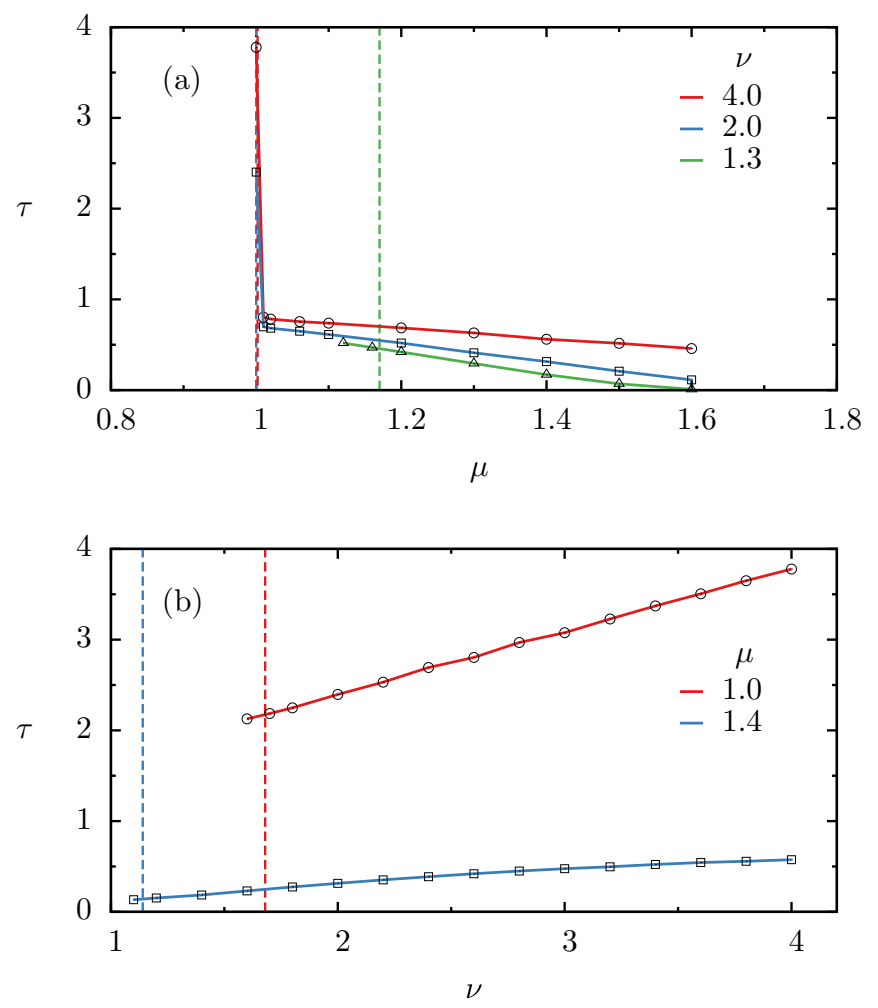

FIG. 3. Characteristic time $\tau$ of the exponential decay of $\rho_{\min }(t) \sim \exp (-t / \tau)$ as a function of (a) $\mu$ (for fixed values of $\nu$ ) and (b) $v$ (for fixed $\mu$ ), as indicated. The dashed vertical lines correspond to the values at which fragmentation occurs as explained in the text.

A phase diagram, depicting the regions in the $\mu-v$ plane where patterns can develop and where they are fragmented or not, is presented in Fig. 5, obtained from numerical simulations. The white region at the left of the vertical solid line corresponds to the values of the exponents for which no patterns arise, in agreement with the condition given by Eq. (3.7), while patterns emerge in the complementary domain. The solid dark area denotes patterns that are fragmented, in the sense defined above.

Fragmentation occurs depending on the balance between diffusion and growth at low densities. Looking at Fig. 5, we see that fragmentation is favored when the diffusion coefficient and per capita reproduction rate increase superlinearly with the population concentration ( $v$ and $\mu$ larger than one). Differently, when $v$ and $\mu$ are small, diffusion and per capita growth diverge at low densities, promoting the fast occupation of unpopulated regions, thus connecting clusters.

More details about the pattern shape transitions are shown in Fig. 6. We see that crossing the frontier of fragmentation along the line $v=4$ [Fig. 6(a)], there is a smooth variation in the shape quantities $\sigma, \Delta$, and $\rho_{\max }$, as in the cases of Fig. 4 (except that as $\mu \rightarrow 2$, nonlinearities affect the number of peaks $m$ and hence the measured quantities). But when $v$ becomes small, the behavior of pattern features changes. In Figs. 6(b) and 6(c), we note a region where quantities that characterize the shape vary exponentially with $\mu$, followed by a regime in which changes occur more rapidly. Note, for example, that while the height of a peak $\rho_{\max }$ rapidly increases, its width $\sigma$ decreases with $\mu$, suggesting that each
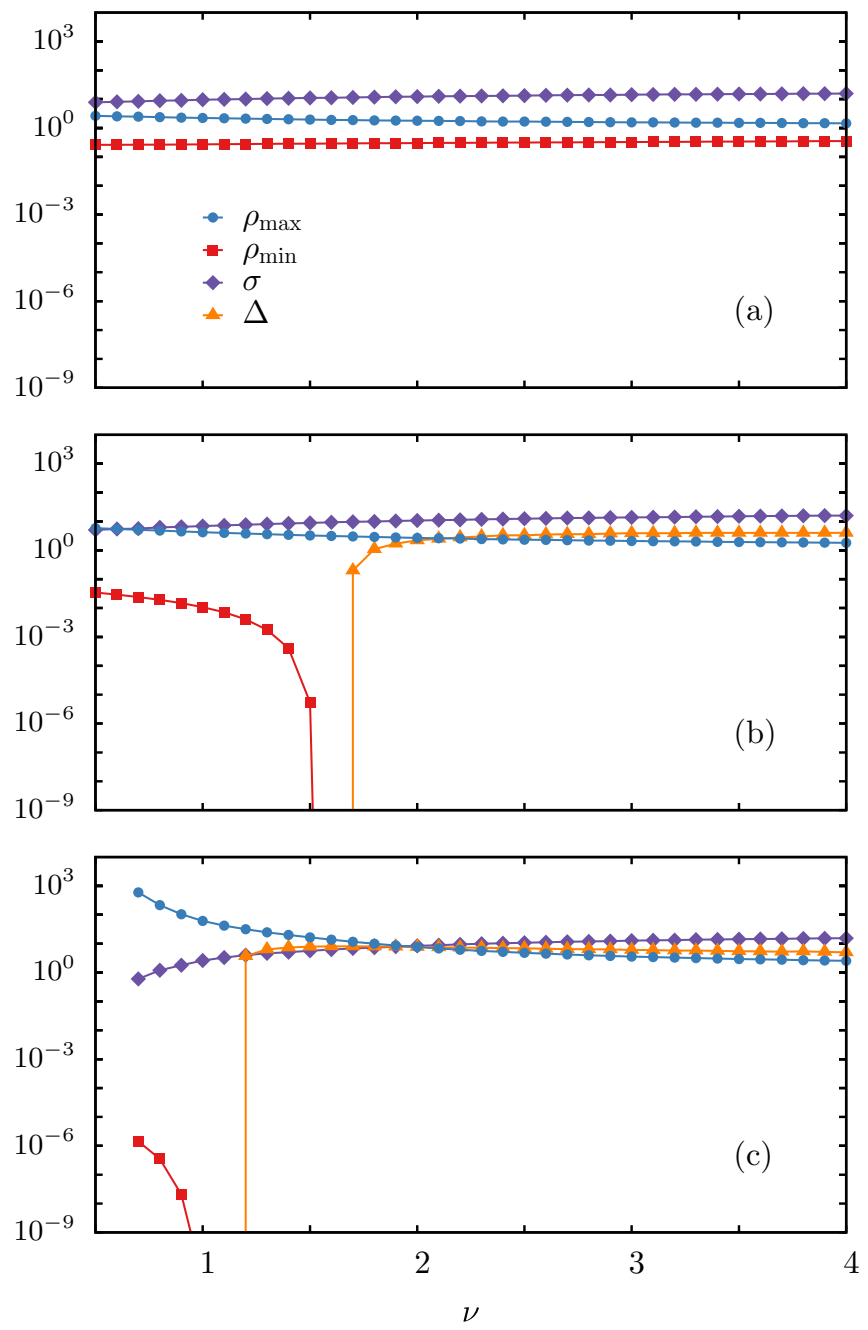

FIG. 4. Stationary values of maximal density $\left(\rho_{\max }\right)$, minimal density $\left(\rho_{\min }\right)$, width at half height $(\sigma)$, and valley width $(\Delta)$ as a function of $v$, for (a) $\mu=0.9$, (b) $\mu=1.0$, and (c) $\mu=1.4$.

peak tends to approach a Dirac delta-like profile. The effect is accentuated for small $v$, as can be seen in Fig. 6(c). Moreover, it causes numerical difficulties, which prevent the determination of whether a Dirac delta is attained or not for finite $\mu$, since the increments $d x$ and $d t$ used in simulations must be reduced, thus increasing the computational cost. It is worth mentioning that, although the dependency of $\rho_{\text {min }}$ with the model exponents is similar to those in Figs. 4(b) and 4(c), mainly the sharp drop, we could not follow the behavior until $\varrho$ is attained (or not) due to strong instability in the numerical integration when $\mu \rightarrow 2$ (hatched region in Fig. 5). Such complications compromise a definite conclusion regarding the fragmentation process for large values of $\mu$, and especially for small $v$.

Finally, concerning the timescales of the pattern shape transition, we address further comments related to Fig. 3. For large values of $v[v=2.0,4.0$ in Fig. 3(a)], the time $\tau$ explodes as $\mu$ approaches the critical value for fragmentation. In these cases, the characteristic time of relaxation towards a local null population level (when fragmentation occurs) and the time of relaxation towards a finite minimum population (otherwise) suffer a drastic change. That is, together with 


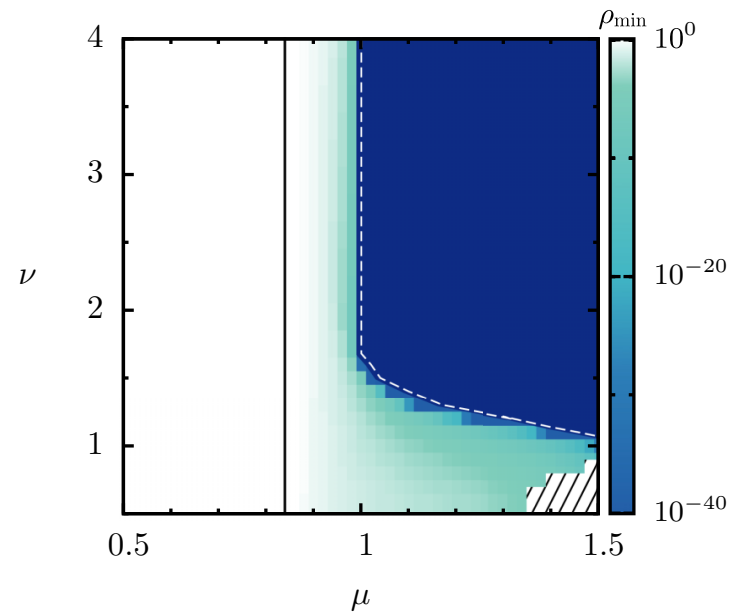

FIG. 5. Phase diagram, in the $\mu-v$ plane. The color scale represents the stationary minimal density $\rho_{\min }$. The vertical solid line at $\mu_{p} \simeq 0.84$ is the onset of pattern formation, according to Eq. (3.7). In that region, $\rho_{\min }=\rho_{\max }=1$. Above $\mu_{p}$ patterns emerge, whose minimal value gradually decreases. The dashed line separates the nonfragmented region in color scale from the fragmented one (solid dark color). The hatched region corresponds to values not calculated due to computational limitations.

the transition related to the minimum value of the stationary density $\rho_{\min }$, there is a transition in the timescale of the dynamics, which becomes slower when $\mu$ approaches the critical point [see Figs. 3(a) and 3(b)]. In contrast, there are other cases where a drastic change in the timescale is not observed, and there is continuity of the values of $\tau$ across the fragmentation boundary; that is, the time of decay of the density towards a finite level (at the left of the vertical lines in the figure) or towards zero (at the right of the vertical lines) does not suffer a discontinuity. This indicates that depending on the region of the $\mu-v$ plane, the transition to fragmentation can occur in two distinct ways.

The relation between nonlinearities and patterns shape and its implication for population dynamics will be discussed in the following section.

\section{SUMMARY AND DISCUSSION}

Using as a starting point a nonlocal Fisher-KPP equation, which became a relevant description in mathematical biology $[38,40-42,49,50]$, we introduce density-dependent feedback in diffusion and growth processes and investigate its effects in shaping the population distribution. We choose the particular form of power-law dependencies on the density that allows us to contemplate a large class of responses to population density, as found in populations of insects, bacteria, and vegetation, among other cases, where diffusion and growth can be either enhanced or harmed by the concentration of individuals.

The emerging patterns have shapes ranging from mild oscillations around a reference level to disconnected clusters. The regulatory mechanisms of population growth, represented by $\mu$, are crucial for the emergence of patterns as well as for fragmentation. The same can be said about the type of
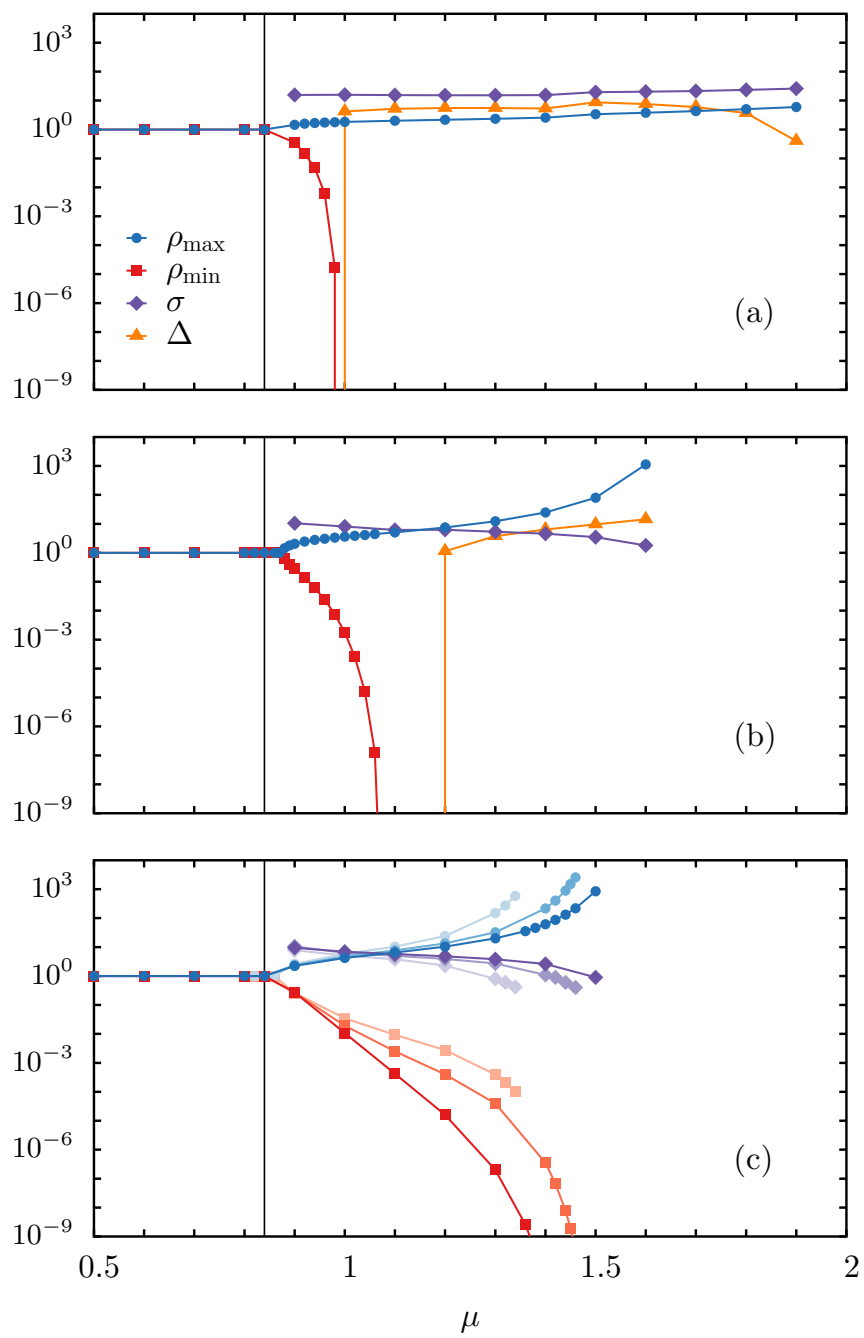

FIG. 6. Stationary values of the maximal density $\left(\rho_{\max }\right)$, minimal density $\left(\rho_{\min }\right)$, width at half height $(\sigma)$, and valley width $(\Delta)$ as a function of $\mu$, for (a) $v=4.0$, (b) $v=1.3$, and (c) $v=0.5,0.8$, and 1.0 , where more intense colors correspond to higher values of $v$. The vertical lines represent $\mu_{p} \simeq 0.84$.

diffusion controlled by $v$, despite that, in general, diffusion has an homogenizing effect.

In particular, we focused on the self-induced population fragmentation, determining the conditions that nonlinearities must obey. Briefly, we observed that fragmentation is favored when growth and diffusion coefficients are positively correlated with population density. Moreover, it arises from a complex interplay between growth and dispersal processes and nonlocality.

Beyond linear stability analysis, our results are supported by numerical simulations. Since Eq. (2.5) is nonlinear and nonlocal, it is difficult to access analytically the features of the stationary solutions. Despite that, analytical solutions can be obtained in very special cases. For instance, neglecting diffusion and considering constant growth rate $(\mu=1)$, for the homogeneous influence function, it is possible to obtain the peaks' shape [43,51]. Regarding the role of densitydependent feedback, insights can be brought from studies outside the context of pattern formation, where the evolution of a single peak follows a similar nonlinear but local 

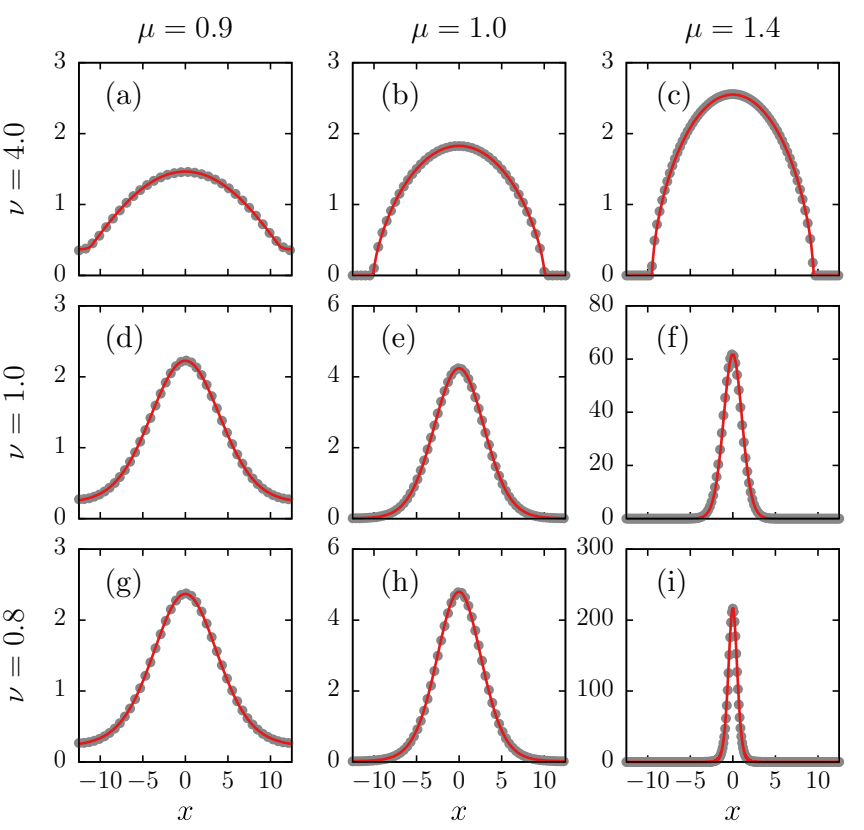

FIG. 7. Stationary profiles obtained numerically together with the description given by Eq. (A1), for the values of $\mu, v$ indicated. Fitting values of the parameters are given in Table I.

equation $[16,36,52,53]$. In these works, the single peak, generated by power-law density dependencies, can have a shape, which can range from concave to sharp, as found in the present context. In particular cases, the exact solutions fall into the class of a generalized Gaussian shape [16,22,52,53]. These previous works motivated us to propose a periodic extension of that ansatz for the profiles shown in Fig. 1, namely, Eq. (A2), which describes remarkably well the numerical patterns (see Fig. 7 in the Appendix). Parameter $\beta$ in Eq. (A1) can be used to characterize pattern shape. Notice that $\beta=0$ corresponds to a Gaussian, while $\beta>0(<0)$ to platykurtic (leptokurtic) clusters. In particular, for $\beta>0$, each individual cluster has compact support. This condition is associated with the emergence of fragmentation, together with the constraint of nonoverlap, $2 x_{0}<\Lambda$, as defined in the Appendix. These two conditions reproduce well the fragmented-patterns region in the phase diagram (Fig. 5), where $\rho_{\text {min }} \rightarrow \varrho$. Let us mention that this phase diagram will change with other choices of the length $\ell$ of the boxlike kernel, as well as of other shapes of the influence kernel, but it qualitatively exhibits the diversity of patterns that Eq. (2.5) can yield.

To establish a connection between the parameters $\{\mu, \nu\}$ in Eq. (2.5) and $\{A, s, \beta\}$ in Eq. (A1) also faces obstacles due to the particular nonlinear and nonlocal character of the dynamics. For instance, standard perturbative approaches can provide sinelike solutions near the transition to patterns (small-amplitude limit). However, these solutions cannot be analytically compared with the ansatz given by Eq. (A2) since it has no closed form in Fourier space. A perturbative treatment would find additional challenges related to the fact that fragmentation occurs far from the transition to patterns.

Regarding the definition of fragmentation, previous models for pattern formation, which helped to explain selforganization in mussels [54], bacteria [55], vegetation under the sea [56], and semiarid ecosystems [37,38], produce an arrangement of high-density clusters interleaved by low-density regions. In some cases, when clusters are sharply defined or well spaced, the population level in between can be very low. More specifically, in these cases, population concentration is expected to decay exponentially as we move away from the peaks (see, for instance, Ref. [37]). Taking into account that a biological population is constituted by a finite number of individuals, the occurrence of very low densities in the meanfield description can be associated with an effective fragmentation of the population. This is because, in the continuous density description, it is possible to emulate the finiteness of the population by means of a threshold value, inversely proportional to the number of individuals and below which the density is considered null. Under this perspective, the region for fragmentation in the phase diagram of Fig. 5 would be effectively enlarged as the number of individuals diminishes. In contrast, according to our model, density-dependent feedback drives the population density between clusters to zero in the long-time limit, such that the stationary profiles are composed of clusters with the compact-support property. As a consequence, actual fragmentation occurs, and it is robust independently of the number of individuals (i.e., the threshold value) considered.

Beyond the nonlocal interactions embedded in the influence function, when there are isolated clusters, individuals are in direct contact only with those within the same cluster. This restricts the propagation of contact processes, such as diseases or information, transferred from one individual to another. Initiating the contagion within an isolated cluster, the affected population would be confined, while, in nonfragmented patterns, the information can percolate to the whole population. In fact, arrangements that emerge solely from the interactions have been shown to bring critical consequences to populations dynamics [7-11]. Furthermore, as widely studied in the context of metapopulations, a fragmented habitat can promote population segregation, which also brings consequences to the stability and diversity of ecosystems [5,6]. In our case, the distinct profiles that emerge from the dynamics are also expected to influence the fate of the population. Therefore, as a perspective of future work, it may be worth studying the coevolution of contact processes and population dynamics ruled by Eq. (2.5).

\section{ACKNOWLEDGMENTS}

This work is partially funded by Brazilian Research Agencies Coordenação de Aperfeiçoamento de Pessoal de Nível Superior (CAPES), Conselho Nacional de Desenvolvimento Científico e Tecnológico (CNPq), Fundação de Amparo à Pesquisa do Estado do Rio de Janeiro (FAPERJ), and the Spanish Research Agency, through grant MDM-2017-0711 from the Maria de Maeztu Program for Units of Excellence in $R \& D$.

\section{APPENDIX: SHAPE OF PATTERNS}

We show in this section that the patterns that emerge from the generalized Fisher-KPP Eq. (2.5) can be described in very good approximation by a periodic extension of a 
TABLE I. Parameter values from the (nonlinear least-square) fitting of Eq. (A2), in the interval $[-\Lambda / 2, \Lambda / 2]$ (with $\Lambda=50$ ), to stationary patterns displayed in Fig. 7, after centering a maximum at $x=0$. The square brackets contain the estimated error in the least significant figure (e.g., the notation 213.0[4] stands for 213.0 \pm 0.4 ).

\begin{tabular}{lcccr}
\hline \hline & & $\mu=0.9$ & $\mu=1.0$ & $\mu=1.4$ \\
\hline$v=4.0$ & $A$ & $1.4645[7]$ & $1.8264[7]$ & $2.5578[6]$ \\
& $\beta$ & $0.793[3]$ & $1.428[3]$ & $1.594[2]$ \\
& $s$ & $8.761[9]$ & $8.521[7]$ & $8.501[4]$ \\
$v=1.0$ & $A$ & $2.222[1]$ & $4.245[2]$ & $61.5[2]$ \\
& $\beta$ & $-0.316[3]$ & $-0.076[2]$ & $-0.11[1]$ \\
& $S$ & $4.149[6]$ & $2.912[3]$ & $1.077[6]$ \\
$v=0.8$ & $A$ & $2.367[2]$ & $4.797[8]$ & $213.0[4]$ \\
& $\beta$ & $-0.395[4]$ & $-0.153[7]$ & $-0.259[8]$ \\
& $S$ & $3.777[9]$ & $2.560[9]$ & $0.472[2]$ \\
\hline \hline
\end{tabular}

generalization of the Gaussian function. Inspired by the form of the solutions of the (nonlinear diffusion or) porous media equation [16] and other related ones [21-23,52,53], we consider the ansatz

$$
f(x)=A\left(1-\frac{\beta x^{2}}{2 s^{2}}\right)_{+}^{1 / \beta},
$$

where $A$ and $s$ are positive constants, and $\beta$ is real. The subindex " + " means null value if the expression between parentheses is nonpositive; that is, $f(x)$ has compact support $\left[-x_{0}, x_{0}\right]$, with $x_{0}=s \sqrt{2 / \beta}$.

If $\beta \rightarrow 0$, Eq. (A1) yields the Gaussian function, otherwise it represents the generalized Gaussian that arises within Tsallis statistics [57].

To describe the steady states observed in our case, we consider the periodic extension of Eq. (A1) with period $\Lambda$ :

$$
f^{\mathrm{ext}}(x)=\sum_{k \in \mathbb{Z}} f(x-k \Lambda)
$$

Figure 7 shows stationary patterns adjusted by Eqs. (A1) and (A2), and Table I shows the values of the fitting parameters. Only one wavelength $\Lambda$ of $\rho(x)$ (between successive minima of $\rho$ ) is ploted in Fig. 8. Notice that the offset observed in some of the cases arises from the superposition given by the periodic extension.
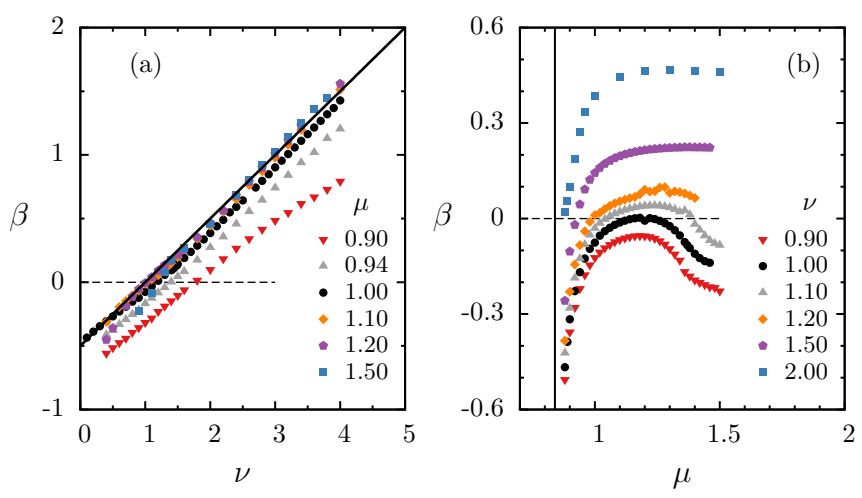

FIG. 8. Ansatz exponent $\beta$ as a function of model exponents $v$ (a), $\mu$ (b). The solid line in panel (a) corresponds to $\beta=(v-1) / 2$, drawn for comparison. The vertical solid line in panel (b) represents $\mu=\mu_{p} \simeq 0.84$. The value $\beta=0$ is highlighted by dashed horizontal lines.

We observe, in Fig. 7 and Table I, that when $v=\mu=1$, the shape is nearly Gaussian, since $\beta \simeq 0$. Gaussian approximations were found for a similar evolution equation with normal diffusion [58]. But when the exponents become different from 1 , deviations from the Gaussian form occur. When $\beta>0(<0)$ $v>1(<1)$, associated with sub(super)-diffusion, clusters are platykurtic (leptokurtic). More importantly, according to Eq. (A1), for $\beta>0$, clusters have the compact-support property (smooth boundary for $0<\beta<1$ and sharp boundary for $\beta>1$ ). This natural cutoff could in principle be associated with fragmentation. But, since clusters are not isolated, there is an additional condition for fragmentation: clusters should not overlap. This condition occurs when the length of the support is shorter than the pattern wavelength, that is, $2 x_{0}<$ $\Lambda$. It is interesting to remark that these conditions match fairly well (not shown) the fragmentation region in the phase diagram of Fig. 5.

The agreement between the ansatz in Eq. (A1) and numerical patterns opens an interesting question regarding the possibility of achieving an, at least approximate, analytical solution of Eq. (2.5), as found for some linear processes [43,51,58]. Nevertheless, from the direct substitution of the periodic ansatz into Eq. (2.5), a straightforward result was not found. Moreover, the relation between the ansatz exponent $\beta$ and the model exponents $\mu, v$ is not evident, but there is a strong trend given by a factor $(v-1) / 2$ [see Fig. 8(a)]. This major contribution to $\beta$ corresponds to the exponent that emerges in the pure nonlinear diffusion [22]. Besides that, the exponent depends also on $\mu$ in a nontrivial way, as can be seen in Fig. 8(b); thus the values of $\beta$ result from the interplay between both processes.
[1] D. Ludwig, D. G. Aronson, and H. F. Weinberger, J. Math. Biol. 8, 217 (1979).

[2] E. M. Baptestini, M. A. de Aguiar, and Y. Bar-Yam, J. Theor. Biol. 335, 51 (2013).

[3] M. G. Turner and R. H. Gardner, Landscape Ecology in Theory and Practice: Pattern and Process (Springer, New York, 2015).
[4] I. Hanski, Metapopulation Ecology, Oxford Series in Ecology and Evolution Vol. 312 (Oxford University Press, Oxford, 1999).

[5] S. A. Levin, Am. Nat. 108, 207 (1974).

[6] L. Fahrig, Ann. Rev. Ecol. Evol. System. 34, 487 (2003).

[7] A. E. Blanchard and T. Lu, BMC Syst. Biol. 9, 59 (2015). 
[8] C. D. Nadell, K. R. Foster, and J. B. Xavier, PLoS Comput. Biol. 6, e1000716 (2010).

[9] O. Hallatschek, P. Hersen, S. Ramanathan, and D. R. Nelson, Proc. Natl. Acad. Sci. USA 104, 19926 (2007).

[10] J.-U. Kreft, Microbiology 150, 2751 (2004).

[11] S. Pigolotti, R. Benzi, M. H. Jensen, and D. R. Nelson, Phys. Rev. Lett. 108, 128102 (2012).

[12] E. H. Colombo and C. Anteneodo, Phys. Rev. E 86, 036215 (2012).

[13] R. Fisher, Ann. Eugenics 7, 355 (1937).

[14] I. G. Petrowsky, Selected Works. Part II, Differential Equations and Probability Theory, edited by O. A. Oleinik, Classics of Soviet Mathematics Series Vol. 5 (Gordon and Breach Publishers, Amsterdam, 1996), pp. 106-132.

[15] J. E Sosa-Hernández, M. Santillán, and J. Santana-Solano, Phys. Rev. E 95, 032404 (2017).

[16] M. Muskat, R. D. Wyckoff et al., Flow of Homogeneous Fluids through Porous Media (McGraw-Hill, New York, 1937).

[17] M. E. Cates, D. Marenduzzo, I. Pagonabarraga, and J. Tailleur, Proc. Natl. Acad. Sci. USA 107, 11715 (2010).

[18] J. D. Murray, Mathematical Biology: I. An Introduction, Interdisciplinary Applied Mathematics Vol. 17 (Springer, New York, 2002).

[19] C. López, Phys. Rev. E 74, 012102 (2006).

[20] V. M. Kenkre and N. Kumar, Proc. Natl. Acad. Sci. USA 105, 18752 (2008).

[21] E. K. Lenzi, C. Anteneodo, and L. Borland, Phys. Rev. E 63, 051109 (2001).

[22] C. Anteneodo, Physica A 358, 289 (2005).

[23] C. Anteneodo, Phys. Rev. E 76, 021102 (2007).

[24] W. I. Newman, J. Theor. Biol. 85, 325 (1980).

[25] M. E. Gurtin and R. C. MacCamy, Math. Biosci. 33, 35 (1977).

[26] P. Kareiva, Oecologia 57, 322 (1983).

[27] G. Birzu, S. Matin, O. Hallatschek, and K. Korolev, bioRxiv (2019), doi:10.1101/565986.

[28] L. S. dos Santos, B. C. Cabella, and A. S. Martinez, Theor. Biosci. 133, 117 (2014).

[29] B. C. T. Cabella, A. S. Martinez, and F. Ribeiro, Phys. Rev. E 83, 061902 (2011).

[30] R. V. dos Santos, F. L. Ribeiro, and A. S. Martinez, J. Theor. Biol. 385, 143 (2015).

[31] B. C. T. Cabella, F. Ribeiro, and A. S. Martinez, Physica A 391, 1281 (2012).

[32] A. S. Martinez, R. S. González, and A. L. Espíndola, Physica A 388, 2922 (2009).

[33] A. S. Martinez, R. S. González, and C. A. S. Terçariol, Physica A 387, 5679 (2008).

[34] F. Courchamp, T. Clutton-Brock, and B. Grenfell, Trends Ecol. Evol. 14, 405 (1999).
[35] M. Walker, A. Hall, R. M. Anderson, and M.-G. Basáñez, Parasites Vectors 2, 11 (2009).

[36] E. Colombo and C. Anteneodo, J. Theor. Biol. 446, 11 (2018).

[37] D. Escaff, C. Fernandez-Oto, M. G. Clerc, and M. Tlidi, Phys. Rev. E 91, 022924 (2015).

[38] C. E. Tarnita, J. A. Bonachela, E. Sheffer, J. A. Guyton, T. C. Coverdale, R. A. Long, and R. M. Pringle, Nature (London) 541, 398 (2017).

[39] C. Fernandez-Oto, O. Tzuk, and E. Meron, Phys. Rev. Lett. 122, 048101 (2019).

[40] M. A. Fuentes, M. N. Kuperman, and V. M. Kenkre, Phys. Rev. Lett. 91, 158104 (2003).

[41] R. Martínez-García, J. M. Calabrese, E. Hernández-García, and C. López, Phil. Trans. R. Soc. A 372, 20140068 (2014).

[42] E. Hernández-García and C. López, Phys. Rev. E 70, 016216 (2004).

[43] S. Pigolotti, C. López, and E. Hernández-García, Phys. Rev. Lett. 98, 258101 (2007).

[44] M. Doebeli and U. Dieckmann, Am. Nat. 156, S77 (2000).

[45] M. Cross and P. Hohenberg, Rev. Mod. Phys. 65, 851 (1993).

[46] W. H. Press, S. A. Teukolsky, W. T. Vetterling, and B. P. Flannery, Numerical Recipes 3rd Edition: The Art of Scientific Computing, 3rd ed. (Cambridge University Press, New York, 2007).

[47] The choice was made to have a relative error smaller than $1 \%$, according to a convergence analysis.

[48] We identify this region as zero density, since within it the null state is numerically stable. Specifically, by zeroing the density values, they remain stable.

[49] C. Fernandez-Oto, M. Tlidi, D. Escaff, and M. Clerc, Phil. Trans. R. Soc. A 372, 20140009 (2014).

[50] J. A. R. da Cunha, A. L. A. Penna, and F. A. Oliveira, Phys. Rev. E 83, 015201(R) (2011).

[51] E. Hernández-García, C. López, S. Pigolotti, and K. H. Andersen, Phil. Trans. R. Soc. A 367, 3183 (2009).

[52] C. Tsallis and D. J. Bukman, Phys. Rev. E 54, R2197 (1996).

[53] P. Troncoso, O. Fierro, S. Curilef, and A. Plastino, Physica A 375, 457 (2007).

[54] J. Van de Koppel, J. C. Gascoigne, G. Theraulaz, M. Rietkerk, W. M. Mooij, and P. M. Herman, Science 322, 739 (2008).

[55] E. Ben-Jacob, O. Schochet, A. Tenenbaum, I. Cohen, A. Czirok, and T. Vicsek, Nature (London) 368, 46 (1994).

[56] D. Ruiz-Reynés, D. Gomila, T. Sintes, E. Hernández-García, N. Marbà, and C. M. Duarte, Sci. Adv. 3, e1603262 (2017).

[57] C. Tsallis, Introduction to Nonextensive Statistical Mechanics: Approaching a Complex World (Springer Science and Business Media, New York, 2009).

[58] F. V. Barbosa, A. A. Penna, R. M. Ferreira, K. L. Novais, J. A. da Cunha, and F. A. Oliveira, Physica A 473, 301 (2017). 